\title{
Investigation of the cytotoxicity of bioinspired coumarin analogues towards human breast cancer cells
}

\author{
Leonidas Gkionis ${ }^{1} \cdot$ Eleni Kavetsou $^{2} \cdot$ Alexandros Kalospyros $^{2} \cdot$ Dimitris Manousakis $^{2} \cdot$ Miguel Garzon Sanz $^{1}$. \\ Sam Butterworth ${ }^{1,3} \cdot$ Anastasia Detsi $^{2} \cdot$ Annalisa Tirella $^{1,3}$
}

Received: 7 February 2020 / Accepted: 1 April 2020 / Published online: 23 April 2020

(c) The Author(s) 2020

\begin{abstract}
Coumarins possess a wide array of therapeutic capabilities, but often with unclear mechanism of action. We tested a small library of 18 coumarin derivatives against human invasive breast ductal carcinoma cells with the capacity of each compound to inhibit cell proliferation scored, and the most potent coumarin analogues selected for further studies. Interestingly, the presence of two prenyloxy groups (5,7-diprenyloxy-4-methyl-coumarin, $\mathbf{4 g}$ ) or the presence of octyloxy substituent (coumarin 4d) was found to increase the potency of compounds in breast cancer cells, but not against healthy human fibroblasts. The activity of potent compounds on breast cancer cells cultured more similarly to the conditions of the tumour microenvironment was also investigated, and increased toxicity was observed. Results suggest that tested coumarin derivatives could potentially reduce the growth of tumour mass. Moreover, their use as (combination) therapy in cancer treatment might have the potential of causing limited side effects.
\end{abstract}

Leonidas Gkionis and Eleni Kavetsou: Co-first authors.

Electronic supplementary material The online version of this article (https://doi.org/10.1007/s11030-020-10082-6) contains supplementary material, which is available to authorized users.

Anastasia Detsi

adetsi@chemeng.ntua.gr

$\triangle$ Annalisa Tirella

annalisa.tirella@manchester.ac.uk

1 Division of Pharmacy and Optometry, Faculty of Biology,

Medicine and Health, Manchester Academic Health

Science Centre, University of Manchester, Oxford Road,

Manchester M13 9PL, UK

2 Laboratory of Organic Chemistry, School of Chemical Engineering, National Technical University of Athens, Heroon Polytechniou 9, Zografou Campus, 15780 Athens, Greece

3 NorthWest Centre for Advanced Drug Delivery (NoWCADD), Faculty of Biology, Medicine and Health, University of Manchester, Oxford Road, Manchester M13 9PT, UK 


\section{Graphic abstract}

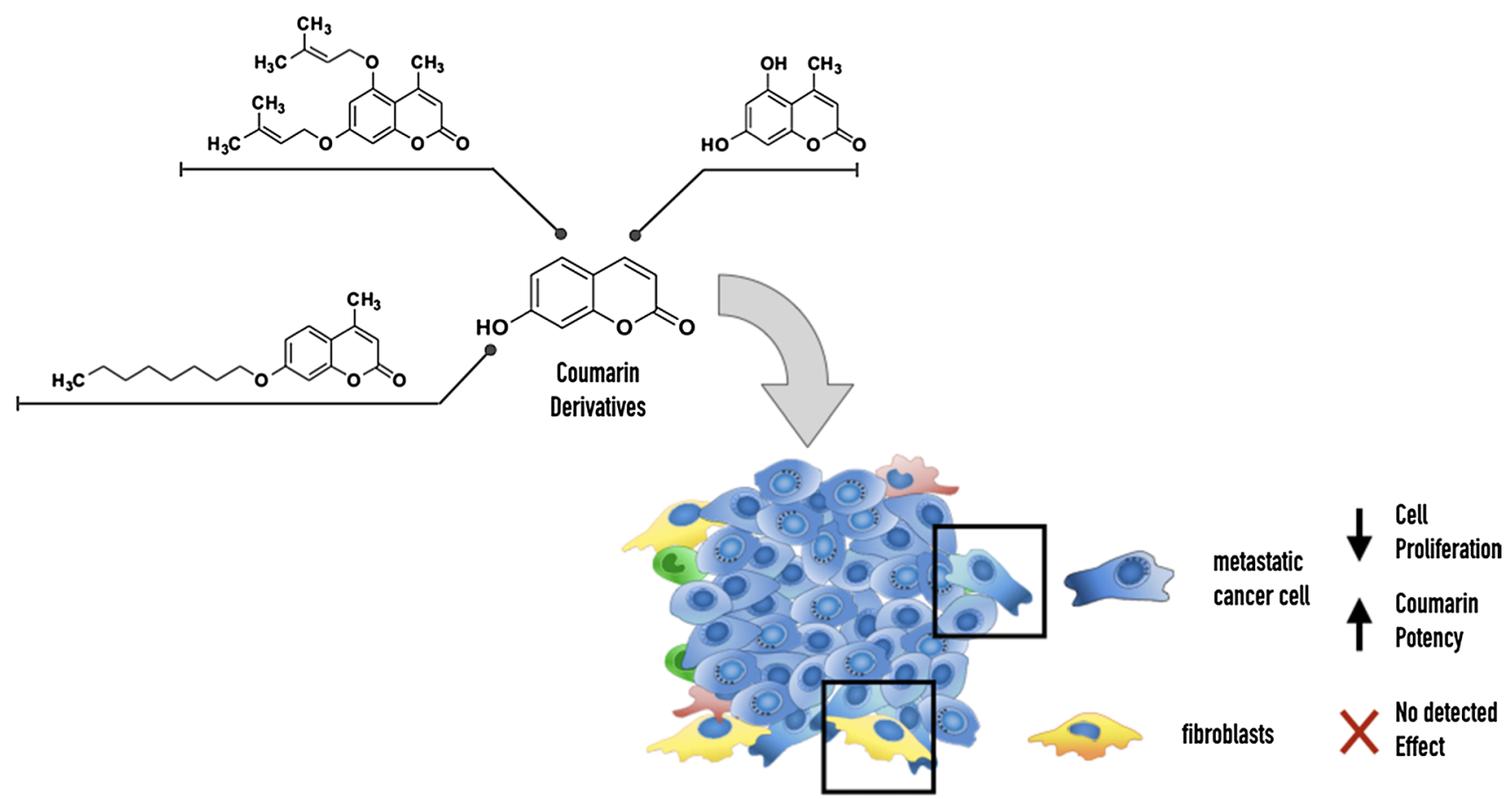

Keywords Alkoxy-coumarins $\cdot$ Auraptene $\cdot$ Umbelliprenin $\cdot$ Breast cancer

\section{Introduction}

Breast carcinoma is considered the predominant and more common malignancy in women worldwide, with one in eight women potentially developing breast cancer during their lifetime [1] and predictions of 3.2 million newly diagnosed cases per year by 2050 [2]. Early detection and intervention are essential to increase patients' survival rate, yet the treatment of advanced cancer remains an issue. While many biological and physicochemical factors have been identified in cancer development, there is an increasing interest in the role of inflammation and involvement of stromal component of the tumour microenvironment [3]. The challenge of treating breast cancer resides not only in the identification of active compounds capable of targeting the cancer, but mainly in identifying potent therapies with low side effects [4]. In this perspective, natural compounds like coumarins have gained significant interest in the recent years for their numerous pharmacological activities including chemopreventive and antiproliferative properties against various cancer types [5-8].

Coumarins are heterocyclic organic compounds that are widely distributed in nature $[4,9]$. Coumarin derivatives have gained high scientific interest as promising drug candidates since they possess multiple pharmacological properties [10-13], such as antioxidant [14-16], antibacterial [17, 18], antimicrobial [18], antiviral [13], hepatoprotective [19] and anti-inflammatory effects [20-22]. Natural and synthetic coumarins have been also reported as effective chemopreventive and anticancer agents in vitro [23-26] and in vivo [27].

Chemical modification such as alkylation (the addition of unsaturated or saturated chain to the coumarin scaffold) has been shown to enhance the pharmacological profile of several coumarins, especially their anticancer activity [25]. In particular, the insertion of an unsaturated chain (prenyl, geranyl or farnesyl side chain) is known as prenylation and constitutes a metabolic pathway in nature (including plant kingdom and microorganism such as fungi and bacteria [28]). The process of prenylation is considered to further enhance the pharmacological activity of these metabolites mostly because it strengthens the lipophilicity of the molecules [29]. Recently, natural oxyprenylated coumarins (isopentenyloxy (C5), geranyloxy (C10) and farnesyloxy (C15) compounds and their biosynthetic derivatives) have been studied for their pharmacological properties [28], mainly as potential anticancer agents [30]. Auraptene (7-geranyloxy coumarin) and umbelliprenin are the most common plantderived oxyprenylated coumarins, first isolated from citrus fruits and Ferula plant species, respectively, and present a wide range of bioactivities [25, 31-35]. The addition of an 
aliphatic chain to the coumarin scaffold is another modification shown to have anticancer effects as reported by Farley et al. [25], who reported that octyloxy-coumarins possess cytotoxicity against pancreatic cancer cells with concentrations in the order of tens of nM. As a continuation to our previous work concerning the biological evaluation of structurally modified coumarin analogues [14, 15], a series of bioinspired synthetic alkoxy coumarin derivatives (bearing saturated and unsaturated chains) were synthesized, structurally characterized and evaluated for their cytotoxicity against breast cancer cell lines (MCF-7 and MDA-MB-231) and fibroblasts. Interestingly we found that the more potent coumarin compounds have no effect on fibroblast (off-target control) and increase their potency on breast cancer cells cultured under nutrient-deprived conditions similar to the tumour microenvironment.

\section{Materials and methods}

\section{Coumarins analogues}

\section{Synthesis}

The chemicals used for synthesis and analysis were purchased from Sigma-Aldrich or Alfa Aesar (7-hydroxycoumarin, 98\%) and used without further purification. NMR spectra were recorded on a Varian $300 \mathrm{MHz}$ and $600 \mathrm{MHz}$ spectrometer at the Institute of Chemical Biology of the National Hellenic Research Foundation. The HRMS spectra were obtained using a UHPLC-MSn Orbitrap Velos-Thermo mass spectrometer. Melting points were determined on a Gallenkamp MFB-595 melting point apparatus and are uncorrected.

General procedure for the synthesis of hydroxy or dihydroxy-4-substituted coumarin analogues The desired compounds $\mathbf{3 a}$ and $\mathbf{3 b}$ were synthesized according to the method of Prousis et al. [36].

7-Hydroxy-4-propyl-2H-chromen-2-one (3a) Beige solid; yield $80 \%$ (735.4 mg, $3.60 \mathrm{mmol}$ ); mp $130{ }^{\circ} \mathrm{C}$ (lit. m.p. $\left.127-128{ }^{\circ} \mathrm{C}\right)$ [36]. ${ }^{1} \mathrm{H}$ NMR $\left(600 \mathrm{MHz}\right.$, DMSO- $\left.d_{6}\right)$ : $\delta(\mathrm{ppm}) 10.51(\mathrm{~s}, 1 \mathrm{H}, 7-\mathrm{OH}), 7.64(\mathrm{~d}, J=9.0 \mathrm{~Hz}, 1 \mathrm{H}, \mathrm{H}-5)$, 6.79 (d, $J=8.4 \mathrm{~Hz}, 1 \mathrm{H}, \mathrm{H}-6), 6.71$ (s, 1H, H-8), 6.08 (s, 1H, $\mathrm{H}-3), 2.70$ (t, $\left.J=7.5 \mathrm{~Hz}, 2 \mathrm{H}, 4-\mathrm{CH}_{2} \mathrm{CH}_{2} \mathrm{CH}_{3}\right), 1.62(\mathrm{~m}, 2 \mathrm{H}$, 4- $\mathrm{CH}_{2} \mathrm{CH}_{2} \mathrm{CH}_{3}$ ), 0.96 (t, $J=7.2 \mathrm{~Hz}, 3 \mathrm{H}, 4-\mathrm{CH}_{2} \mathrm{CH}_{2} \boldsymbol{C H}_{3}$ ).
5,7-Dihydroxy-4-methyl-2H-chromen-2-one (3b) Beige solid; yield $93 \%$ (707.2 mg, $3.68 \mathrm{mmol}$ ); $\mathrm{mp} 288-289{ }^{\circ} \mathrm{C}$ (lit. m.p. 289-290 $\left.{ }^{\circ} \mathrm{C}\right)$ [36]. ${ }^{1} \mathrm{H}$ NMR (600 MHz, DMSO$\left.d_{6}\right): \delta(\mathrm{ppm}) 10.51(\mathrm{~s}, 1 \mathrm{H}, 7-\mathrm{OH}), 10.29(\mathrm{~s}, 1 \mathrm{H}, 5-\mathrm{OH}), 6.25$ (s,1H, H-8), 6.16 (s, 1H, H-6), 5.84 (s, 1H, H-3), 2.48 (d, $\left.J=9.6 \mathrm{~Hz}, 3 \mathrm{H}, 4-\mathrm{CH}_{3}\right)$.

Synthesis of geranylgeranyl iodide The following method was adapted from Alvarez-Manzaneda et al. [37]; briefly, $1170.0 \mathrm{mg}$ (1.72 mmol, 1 eq.) of imidazole and $450.0 \mathrm{mg}$ (1.72 mmol, 1 eq.) of triphenylphosphine were dissolved in $10 \mathrm{~mL}$ of anhydrous dichloromethane (DCM) in a roundbottom flask. $435.0 \mathrm{mg}(1.72 \mathrm{~mol}, 1$ eq. $)$ of iodine was added slowly, and the mixture was stirred for $30 \mathrm{~min}$. Then, the flask was covered with aluminium foil and placed in an ice bath, followed by slow addition of $0.57 \mathrm{~mL}(1.72 \mathrm{~mol}$, 1 eq.) of geranylgeranyl. The mixture was stirred for approximately $2 \mathrm{~h}$. After the reaction was complete (monitored by TLC in pure hexane), the mixture was filtered through a plug of silica, which was then washed with pure hexane. The solvent was evaporated in vacuo, resulting in a dark oily film. Yield 52\% (51.9 mg).

General procedure for the synthesis of alkoxy-coumarins 4a-4m One eq. of the hydroxy- or dihydroxy-4-substituted coumarins, $\mathbf{3 a - 3 \mathbf { c }}$, and 1 eq. of potassium carbonate $\left(\mathrm{K}_{2} \mathrm{CO}_{3}\right)$ were dissolved in dry acetone. Then, $1.2 \mathrm{eq}$. of the appropriate alkoxy-bromide or geranylgeranyl iodide was added dropwise at room temperature, and the mixture was refluxed for $6 \mathrm{~h}$. After the completion of the reaction, $\mathrm{K}_{2} \mathrm{CO}_{3}$ was filtrated, the precipitate was washed with acetone and the solvent was removed in vacuo. The desired products were purified via silica gel column chromatography in a solvent system of petroleum ether/ethyl acetate (9:1). Diprenyloxy coumarins were obtained in high purity after two steps of silica gel chromatography.

7-Prenyloxy-4-methyl-2H-chromen-2-one (4a) White solid; yield 70\% (61.1 mg, $0.25 \mathrm{mmol}) ; \mathrm{mp} 84{ }^{\circ} \mathrm{C}$ (lit. m.p. $\left.84-86{ }^{\circ} \mathrm{C}\right)[38] .{ }^{1} \mathrm{H}$ NMR $\left(300 \mathrm{MHz}, \mathrm{CDCl}_{3}\right): \delta(\mathrm{ppm}) 7.48$ $(\mathrm{d}, J=8.7 \mathrm{~Hz}, 1 \mathrm{H}, \mathrm{H}-5), 6.86(\mathrm{dd}, J=8.7 \mathrm{~Hz} \& J=2.4 \mathrm{~Hz}$, $1 \mathrm{H}, \mathrm{H}-6), 6.82(\mathrm{~d}, J=2.4 \mathrm{~Hz}, 1 \mathrm{H}, \mathrm{H}-8), 5.48(\mathrm{t}, J=6.9 \mathrm{~Hz}$, $\left.1 \mathrm{H}, \mathrm{H}-2^{\prime}\right), 4.59$ (d, $\left.J=6.9 \mathrm{~Hz}, 2 \mathrm{H}, \mathrm{H}-1^{\prime}\right), 2.42$ (s, 3H, 4- $\mathrm{CH}_{3}$ ), $1.83\left(\mathrm{~s}, 3 \mathrm{H}, 3^{\prime}-\mathrm{CH}_{3}\right), 1.79$ (s, 3H, 4'-- $\left.\mathrm{CH}_{3}\right)$.

7-Geranyloxy-4-methyl-2H-chromen-2-one (4b) [14] Brown gummy solid; yield 68\% (296.8 mg, $0.95 \mathrm{mmol})$. ${ }^{1} \mathrm{H}$ NMR (300 MHz, DMSO- $d_{6}$ ): $\delta(\mathrm{ppm}) 7.64(\mathrm{~d}, J=8.4 \mathrm{~Hz}$, 
1H, H-5), 6.94-6.91 (m, 2H, H-6 \& H-8), 6.18 (s, 1H, H-3), $5.42\left(\mathrm{t}, J=6.0 \mathrm{~Hz}, 1 \mathrm{H}, \mathrm{H}-2^{\prime}\right), 5.03\left(\mathrm{br}, 1 \mathrm{H}, \mathrm{H}-6^{\prime}\right), 4.65(\mathrm{~d}$, $\left.J=6.6 \mathrm{~Hz}, 2 \mathrm{H}, \mathrm{H}-1^{\prime}\right), 2.39$ (s, 3H, 4- $\left.\mathrm{CH}_{3}\right), 2.08-2.06$ (m, $4 \mathrm{H}, \mathrm{H}-4^{\prime}$ \& H-5'), 1.73 (s, 3H, 3'- $\mathrm{CH}_{3}$ ), 1.61 (s, 3H, 8'- $\mathrm{CH}_{3}$ ), $1.56\left(\mathrm{~s}, 3 \mathrm{H}, 7^{\prime}-\mathrm{CH}_{3}\right)$.

7-Farnesyloxy-4-methyl-2H-chromen-2-one (4c) Yellow gummy solid; yield $60 \%$ (319.6 mg, $0.84 \mathrm{mmol}) .{ }^{1} \mathrm{H}$ NMR $\left(300 \mathrm{MHz}, \mathrm{CDCl}_{3}\right): \delta(\mathrm{ppm}) 7.48(\mathrm{~d}, J=8.7 \mathrm{~Hz}, 1 \mathrm{H}$, H-5), 6.86 (dd, $J=8.7 \mathrm{~Hz} \& J=1.8 \mathrm{~Hz}, 1 \mathrm{H}, \mathrm{H}-6), 6.82$ (d, $J=1.8 \mathrm{~Hz}, 1 \mathrm{H}, \mathrm{H}-8), 6.13$ (s, $1 \mathrm{H}, \mathrm{H}-3), 5.47$ (t, $J=6.9 \mathrm{~Hz}$, $\left.1 \mathrm{H}, \mathrm{H}-2^{\prime}\right), 5.10-5.07$ (m, 1H, H-6'), 4.60 (d, $J=6.6 \mathrm{~Hz}, 1 \mathrm{H}$, H-1'), 2.39 (s, 3H, 4-CH $\left.\mathrm{CH}_{3}\right), 2.12-1.95$ (m, 8H, H-4' \& H-5' \& $\left.\mathrm{H}-8^{\prime} \& \mathrm{H}-9^{\prime}\right), 1.76$ (s, 3H, 3'- $\left.\mathrm{CH}_{3}\right), 1.67$ (s, 3H, 7'- $\left.\mathrm{CH}_{3}\right)$, $1.59\left(\mathrm{~s}, 6 \mathrm{H}, 11^{\prime}-\mathrm{CH}_{3} \& 12^{\prime}-\mathrm{CH}_{3}\right) .{ }^{13} \mathrm{C}$ NMR $(75 \mathrm{MHz}$, DMSO- $\left.d_{6}\right): \delta(\mathrm{ppm}) 162.0,160.6,155.1,153.9,141.5,135.1$, $131.1,126.8,124.5,123.9,119.5,113.5,113.1,111.5$, 101.8, 65.6, 39.7, 39.3, 26.6, 26.0, 25.9, 18.6, 17.9, 16.9, 16.3. HRMS (ESI) calcd for $\mathrm{C}_{25} \mathrm{H}_{32} \mathrm{O}_{3} \mathrm{Na}: m / z: 403.2244$, found: 403.2245 .

4-Methyl-7-octyloxy-2H-chromen-2-one (4d) Pale yellow solid; yield $55 \%$ (180 mg, $0.62 \mathrm{mmol}) ; \mathrm{mp} 51{ }^{\circ} \mathrm{C}$ (lit. m.p. $\left.48-50{ }^{\circ} \mathrm{C}\right)$ [24]. ${ }^{1} \mathrm{H}$ NMR $(600 \mathrm{MHz}, \mathrm{DMSO})$ : $\delta(\mathrm{ppm}) 8.58(\mathrm{~d}, J=8.4 \mathrm{~Hz}, 1 \mathrm{H}, \mathrm{H}-5), 6.78(\mathrm{dd}, J=9 \mathrm{~Hz} \&$ $J=2.4 \mathrm{~Hz}, 1 \mathrm{H}, \mathrm{H}-6), 6.68(\mathrm{~d}, J=2.4 \mathrm{~Hz}, 1 \mathrm{H}, \mathrm{H}-8), 6.15$ (s, $1 \mathrm{H}, \mathrm{H}-3), 4.06$ (t, $\left.J=6.6 \mathrm{~Hz}, 2 \mathrm{H}, \mathrm{H}-1^{\prime}\right), 2.39$ (s, 3H, 4- $\mathrm{CH}_{3}$ ), 1.72 (m, 2H, H-2'), 1.40 (m, 2H, H-7'), 1.29 (m, 8H, H-3' \& H-5' \& H-4' \& H-6'), $0.86\left(\mathrm{t}, J=6.9 \mathrm{~Hz}, 3 \mathrm{H}, 7^{\prime}-\mathrm{CH}_{3}\right)$.

7-Prenyloxy-4-propyl-2H-chromen-2-one (4e) Yellow solid; yield $60 \%$ (135.0 mg, $0.49 \mathrm{mmol})$; $\mathrm{mp} 89^{\circ} \mathrm{C}$. ${ }^{1} \mathrm{H}$ NMR $\left(600 \mathrm{MHz}, \mathrm{CDCl}_{3}\right): \delta(\mathrm{ppm}) 7.51(\mathrm{~d}, J=8.4 \mathrm{~Hz}$, $1 \mathrm{H}, \mathrm{H}-5), 6.85$ (dd, $J=9.0 \mathrm{~Hz} \& J=2.4 \mathrm{~Hz}, 1 \mathrm{H}, \mathrm{H}-6$ ), $6.82(\mathrm{~d}, J=2.4 \mathrm{~Hz}, 1 \mathrm{H}, \mathrm{H}-8), 6.11(\mathrm{~s}, 1 \mathrm{H}, \mathrm{H}-3), 5.47(\mathrm{t}$, $\left.J=6.9 \mathrm{~Hz}, 1 \mathrm{H}, \mathrm{H}-2^{\prime}\right), 4.57$ (d, $\left.J=7.2 \mathrm{~Hz}, 2 \mathrm{H}, \mathrm{H}-1^{\prime}\right), 2.69$ (t, $\left.J=7.5 \mathrm{~Hz}, 2 \mathrm{H}, 4-\boldsymbol{C H}_{2} \mathrm{CH}_{2} \mathrm{CH}_{3}\right), 1.80\left(\mathrm{~s}, 3 \mathrm{H}, 3^{\prime}-\mathrm{CH}_{3}\right), 1.76$ (s, 3H, 4'- $\mathrm{CH}_{3}$ ), 1.72-1.69 (m, 2H, 4- $\mathrm{CH}_{2} \boldsymbol{C H}_{2} \mathrm{CH}_{3}$ ), 1.04 $\left(\mathrm{t}, J=7.5 \mathrm{~Hz}, 3 \mathrm{H}, 4-\mathrm{CH}_{2} \mathrm{CH}_{2} \boldsymbol{C H}_{3}\right) .{ }^{13} \mathrm{C}$ NMR $(150 \mathrm{MHz}$, $\left.\mathrm{CDCl}_{3}\right): \delta(\mathrm{ppm}) 161.9,161.7,156.5,155.6,139.3,125.4$, 118.8, 113.0, 112.9, 110.9, 101.8, 77.6, 77.2, 76.7, 65.5, 33.9, 25.9, 21.6, 18.4, 14.1. HRMS (ESI) calcd for $\mathrm{C}_{17} \mathrm{H}_{21} \mathrm{O}_{3}$ $(\mathrm{M}+\mathrm{H})^{+}: m / z: 273.1485$, found: 273.1485 .

7-Octyloxy-4-propyl-2H-chromen-2-one (4f) Beige solid; yield $60 \%$ (350.0 mg, $1.11 \mathrm{mmol}) ; \mathrm{mp} 47{ }^{\circ} \mathrm{C} .{ }^{1} \mathrm{H}$ NMR $\left(600 \mathrm{MHz}, \mathrm{CDCl}_{3}\right): \delta(\mathrm{ppm}) 7.51(\mathrm{~d}, J=9.0 \mathrm{~Hz}, 1 \mathrm{H}$, H-5), 6.84 (dd, $J=8.4 \mathrm{~Hz} \& J=1.8 \mathrm{~Hz}, 1 \mathrm{H}, \mathrm{H}-6), 6.81$ (d, $J=2.4 \mathrm{~Hz}, 1 \mathrm{H}, \mathrm{H}-8), 6.12$ (s, 1H, H-3), 4.01 (t, $J=6.6 \mathrm{~Hz}$, $\left.2 \mathrm{H}, \mathrm{H}-1^{\prime}\right), 2.71$ (t, $\left.J=7.5 \mathrm{~Hz}, 2 \mathrm{H}, 4-\mathrm{CH}_{2} \mathrm{CH}_{2} \mathrm{CH}_{3}\right), 1.80$ (m, $\left.2 \mathrm{H}, \mathrm{H}-2^{\prime}\right), 1.73$ (m, 2H, 4- $\left.\mathrm{CH}_{2} \boldsymbol{C H}_{2} \mathrm{CH}_{3}\right), 1.45$ (m, 2H, H-7'), 1.31 (m, 8H, H-3' \& H-5' \& H-4' \& H-6'), 1.04 (t, $J=7.2 \mathrm{~Hz}$, $\left.3 \mathrm{H}, 4-\mathrm{CH}_{2} \mathrm{CH}_{2} \boldsymbol{C H}_{3}\right), 0.88$ (t, $\left.J=6.9 \mathrm{~Hz}, 3 \mathrm{H}, 7^{\prime}-\mathrm{CH}_{3}\right) \cdot{ }^{13} \mathrm{C}$ NMR $\left(150 \mathrm{MHz}, \mathrm{CDCl}_{3}\right): \delta(\mathrm{ppm}) 162.2,161.8,156.6$, $155.7,125.4,112.9,110.8,101.6,68.8,33.9,31.9,29.4$, 29.3, 29.1, 26.1, 22.8, 21.7, 14.2, 14.1. HRMS (APCI) calcd for $\mathrm{C}_{20} \mathrm{H}_{29} \mathrm{O}_{3}(\mathrm{M}+\mathrm{H})^{+}: \mathrm{m} / z: 317.2116$, found: 317.2105.

5,7-Diprenyloxy-4-methyl-2H-chromen-2-one (4 g) Green solid; yield 40\% (272.6 mg, $0.83 \mathrm{mmol}$ ); mp $90{ }^{\circ} \mathrm{C} .{ }^{1} \mathrm{H}$ NMR $\left(600 \mathrm{MHz}, \mathrm{CDCl}_{3}\right): \delta(\mathrm{ppm}) 6.43(\mathrm{~d}$, $J=2.4 \mathrm{~Hz}, 1 \mathrm{H}, \mathrm{H}-6), 6.31$ (d, $J=1.8 \mathrm{~Hz}, 1 \mathrm{H}, \mathrm{H}-8), 5.93$ (d, $J=0.6 \mathrm{~Hz}, 1 \mathrm{H}, \mathrm{H}-3$ ), 5.47 (pseudotriplet, $2 \mathrm{H}, \mathrm{H}-2^{\prime}$ \& H-2"), 4.53 (dd, $\left.J=7.2 \mathrm{~Hz} \& J=9.6 \mathrm{~Hz}, 4 \mathrm{H}, \mathrm{H}-1^{\prime} \& \mathrm{H}-1^{\prime \prime}\right), 2.53$ (s, $\left.3 \mathrm{H}, 4-\mathrm{CH}_{3}\right), 1.81$ (s, 3H, 3'- $\left.\mathrm{CH}_{3}\right), 1.80\left(\mathrm{~s}, 3 \mathrm{H}, 4^{\prime}-\mathrm{CH}_{3}\right), 1.76$ (s, 3H, 3"--CH $), 1.73$ (s, 3H, 4"-- $\left.\mathrm{CH}_{3}\right) \cdot{ }^{13} \mathrm{C}$ NMR $(150 \mathrm{MHz}$, $\left.\mathrm{CDCl}_{3}\right): \delta(\mathrm{ppm}) 162.1,161.4,158.4,157.1,154.8,139.4$, 138.7, 118.8, 111.3, 105.1, 96.9, 94.1, 65.9, 65.4, 25.9, 25.8, 24.5, 18.4, 18.3. HRMS (ESI) calcd for $\mathrm{C}_{20} \mathrm{H}_{24} \mathrm{O}_{4}(\mathrm{M}+\mathrm{H})^{+}$: $m / z: 351.1567$, found: 351.1561 .

\section{5,7-Diprenyloxy-4-propyl-2H-chromen-2-one} (4 h) White solid; yield $42 \%$ (272.5 mg, $0.76 \mathrm{mmol})$; $\mathrm{mp}$ 71-72 ${ }^{\circ} \mathrm{C} .{ }^{1} \mathrm{H}$ NMR $\left(600 \mathrm{MHz}, \mathrm{CDCl}_{3}\right): \delta(\mathrm{ppm}) 6.44(\mathrm{~d}$, $J=2.4 \mathrm{~Hz}, 1 \mathrm{H}, \mathrm{H}-6), 6.32$ (d, $J=1.8 \mathrm{~Hz}, 1 \mathrm{H}, \mathrm{H}-8), 5.94$ (s, $1 \mathrm{H}, \mathrm{H}-3$ ), 5.50 (t, $\left.J=7.2 \mathrm{~Hz}, 1 \mathrm{H}, \mathrm{H}-2^{\prime \prime}\right), 5.47$ (t, $J=6.0 \mathrm{~Hz}$, $\left.1 \mathrm{H}, \mathrm{H}-2^{\prime \prime}\right), 4.54$ (d, $\left.J=6.6 \mathrm{~Hz}, 2 \mathrm{H}, \mathrm{H}-1^{\prime}\right), 4.51$ (d, $J=6.6 \mathrm{~Hz}$, 2H, H-1"), 2.84 (t, J=7.8 Hz, 2H, 4- $\boldsymbol{C H}_{2} \mathrm{CH}_{2} \mathrm{CH}_{3}$ ), 1.81 (s, 6H, 3'- $\left.\mathrm{CH}_{3} \& 4^{\prime}-\mathrm{CH}_{3}\right), 1.76\left(\mathrm{~s}, 3 \mathrm{H}, 3^{\prime \prime}-\mathrm{CH}_{3}\right), 1.74$ (s, $\left.3 \mathrm{H}, 4^{\prime \prime}-\mathrm{CH}_{3}\right), 1.61-1.57\left(\mathrm{~m}, 2 \mathrm{H}, 4-\mathrm{CH}_{2} \boldsymbol{C H}_{2} \mathrm{CH}_{3}\right), 0.97$ $\left(\mathrm{t}, J=7.8 \mathrm{~Hz}, 3 \mathrm{H}, 4-\mathrm{CH}_{2} \mathrm{CH}_{2} \boldsymbol{C H}_{3}\right) .{ }^{13} \mathrm{C}$ NMR $(150 \mathrm{MHz}$, $\left.\mathrm{CDCl}_{3}\right): \delta(\mathrm{ppm}) 161.9,161.5,158.6,158.0,157.4,139.4$, 139.3, 118.8, 118.6, 110.8, 104.4, 96.9, 94.3, 65.7, 65.4, 38.8, 25.9, 25.8, 23.4, 18.4, 18.3, 13.9. HRMS (ESI) calcd for $\mathrm{C}_{22} \mathrm{H}_{28} \mathrm{O}_{4}(\mathrm{M}+\mathrm{H})^{+}: \mathrm{m} / \mathrm{z}$ : 379.1880 , found: 379.1869 .

\section{5,7-Digeranyloxy-4-propyl-2H-chromen-2-one}

(4i) White solid; yield $41 \%$ (262.6 mg, $0.53 \mathrm{mmol})$; $\mathrm{mp}$ $79{ }^{\circ} \mathrm{C} .{ }^{1} \mathrm{H}$ NMR $\left(600 \mathrm{MHz}, \mathrm{CDCl}_{3}\right): \delta(\mathrm{ppm}) 6.45(\mathrm{~d}$, $J=1.8 \mathrm{~Hz}, 1 \mathrm{H}, \mathrm{H}-6), 6.33$ (d, $J=1.8 \mathrm{~Hz}, 1 \mathrm{H}, \mathrm{H}-8), 5.95$ (s, $1 \mathrm{H}, \mathrm{H}-3), 5.51$ (t, $\left.J=6.6 \mathrm{~Hz}, 1 \mathrm{H}, \mathrm{H}-1^{\prime}\right), 5.46$ (t, $J=6.6 \mathrm{~Hz}$, 1H, H-1"), 5.09 (pseudotriplet, 2H, H-6' \& H-6"), 4.57 (d, $\left.J=6.6 \mathrm{~Hz}, 2 \mathrm{H}, \mathrm{H}-2^{\prime}\right), 4.54$ (d, $\left.J=6.6 \mathrm{~Hz}, 2 \mathrm{H}, \mathrm{H}-2^{\prime \prime}\right), 2.85$ (t, $\left.J=7.8 \mathrm{~Hz}, 2 \mathrm{H}, 4-\mathrm{CH}_{2} \mathrm{CH}_{2} \mathrm{CH}_{3}\right), 2.13-2.09(\mathrm{~m}, 8 \mathrm{H}$, H-4', H-5' \& H-4", H-5"), 1.76 (s, 3H, 3'- $\mathrm{CH}_{3}$ ), 1.73 (s, $\left.3 \mathrm{H}, 3^{\prime \prime}-\mathrm{CH}_{3}\right), 1.68$ (s, 3H, 7'- $\left.\mathrm{CH}_{3}\right), 1.67$ (s, 3H, 8'- $\mathrm{CH}_{3}$ ), $1.61\left(\mathrm{~s}, 6 \mathrm{H}, 7 "-\mathrm{CH}_{3} \& 8^{\prime \prime}-\mathrm{CH}_{3}\right), 0.98(\mathrm{t}, J=7.8 \mathrm{~Hz}, 3 \mathrm{H}$, $\left.4-\mathrm{CH}_{2} \mathrm{CH}_{2} \boldsymbol{C H}_{3}\right) .{ }^{13} \mathrm{C}$ NMR $\left(150 \mathrm{MHz}, \mathrm{CDCl}_{3}\right): \delta(\mathrm{ppm})$ 162.0, 161.6, 158.6, 158.0, 157.4, 142.5, 142.4, 132.1, 132.0, 123.8, 123.7, 118.6, 118.4, 110.8, 104.4, 96.9, 94.3, 65.8, 65.4, 39.7, 39.6, 38.8, 31.0, 26.4, 26.3, 25.8, 25.7, 23.3, 17.9, 17.8, 16.9, 16.8, 14.1. HRMS (APCI) calcd for $\mathrm{C}_{32} \mathrm{H}_{45} \mathrm{O}_{4}(\mathrm{M}+\mathrm{H})^{+}: \mathrm{m} / \mathrm{z}:$ 493.3312, found: 493.3302 . 
7-Prenyloxy-2H-chromen-2-one (4j) White solid; yield $65 \%$ (59.9 mg, $0.26 \mathrm{mmol}$ ); mp $77{ }^{\circ} \mathrm{C}$ (lit. m.p. $77-78{ }^{\circ} \mathrm{C}$ ) [39]. ${ }^{1} \mathrm{H}$ NMR $\left(300 \mathrm{MHz}, \mathrm{CDCl}_{3}\right): \delta(\mathrm{ppm}) 7.63(\mathrm{~d}$, $J=9.6 \mathrm{~Hz}, 1 \mathrm{H}, \mathrm{H}-4), 7.36$ (d, $J=8.4 \mathrm{~Hz}, 1 \mathrm{H}, \mathrm{H}-5), 6.86-6.82$ (m, 2H, H-6 \& H-8), 6.25 (d, J=9.3 Hz,1H, H-6), 5.48 (t, $\left.J=8.1 \mathrm{~Hz}, 1 \mathrm{H}, \mathrm{H}-2^{\prime}\right), 4.59$ (d, $\left.J=6.9 \mathrm{~Hz}, 2 \mathrm{H}, \mathrm{H}-1^{\prime}\right), 1.84$ (s, $\left.3 \mathrm{H}, 3^{\prime}-\mathrm{CH}_{3}\right), 1.79$ (s, $\left.3 \mathrm{H}, 4^{\prime}-\mathrm{CH}_{3}\right)$.

7-Geranyloxy-2H-chromen-2-one (auraptene) (4k) White solid; yield $60 \%$ (268.5 mg, $0.90 \mathrm{mmol})$; $\mathrm{mp}$ $63{ }^{\circ} \mathrm{C}$ (lit. m.p. 62-63 $\left.{ }^{\circ} \mathrm{C}\right)[40] .{ }^{1} \mathrm{H}$ NMR $\left(300 \mathrm{MHz}, \mathrm{CDCl}_{3}\right)$ : $\delta(\mathrm{ppm}) 7.63(\mathrm{~d}, J=9.3 \mathrm{~Hz}, 1 \mathrm{H}, \mathrm{H}-4), 7.36(\mathrm{~d}, J=8.4 \mathrm{~Hz}, 1 \mathrm{H}$, H-5), 6.86-6.82 (m, 2H, H-6 \& H-8), 6.24 (d, $J=9.3 \mathrm{~Hz}$, $1 \mathrm{H}, \mathrm{H}-3), 5.47\left(\mathrm{t}, J=6 \mathrm{~Hz}, 1 \mathrm{H}, \mathrm{H}-2^{\prime}\right), 5.09(\mathrm{t}, J=5.7 \mathrm{~Hz}$, $\left.1 \mathrm{H}, \mathrm{H}-6^{\prime}\right), 4.62\left(\mathrm{~d}, J=6.6 \mathrm{~Hz}, 2 \mathrm{H}, \mathrm{H}-1^{\prime}\right), 2.15-2.11(\mathrm{~m}, 4 \mathrm{H}$, H-4' \& H-5'), 1.79 (s, 3H, 3'- $\mathrm{CH}_{3}$ ), 1.69 (s, 3H, 8'- $\mathrm{CH}_{3}$ ), $1.63\left(\mathrm{~s}, 3 \mathrm{H}, 7^{\prime}-\mathrm{CH}_{3}\right)$.

7-Farnesyloxy-coumarin (umbelliprenin) (4I) Yellowish solid; yield $80 \%$ (439.8 $\mathrm{mg}, 1.20 \mathrm{mmol}$ ); $\mathrm{mp} 61{ }^{\circ} \mathrm{C}$ (lit. m.p. $\left.58-60{ }^{\circ} \mathrm{C}\right)[40] .{ }^{1} \mathrm{H} \mathrm{NMR}\left(300 \mathrm{MHz}, \mathrm{CDCl}_{3}\right): \delta(\mathrm{ppm})$ $7.62(\mathrm{~d}, J=9.3 \mathrm{~Hz}, 1 \mathrm{H}, \mathrm{H}-4), 7.35(\mathrm{~d}, J=8.1 \mathrm{~Hz}, 1 \mathrm{H}, \mathrm{H}-5)$, 6.86-6.82 (m, 2H, H-6 \& H-8), $6.24(\mathrm{~d}, J=9.6 \mathrm{~Hz}, 1 \mathrm{H}$, H-3), 5.48 (t, $\left.J=6.6 \mathrm{~Hz}, 1 \mathrm{H}, \mathrm{H}-2^{\prime}\right), 4.62(\mathrm{~d}, J=6.6 \mathrm{~Hz}, 1 \mathrm{H}$, H-1'), 2.18 -2.01 (m, 8H, H-4' \& H-5' \& H-8' \& H-9'), 1.79 (s, 3H, 3'-CH3), 1.70 (s, 3H, 8'-CH3), 1.63 (s, 6H, 11'-CH3 $\left.\& 12^{\prime}-\mathrm{CH} 3\right)$.

7-Geranylgeranyloxy-coumarin (4m) [41] Yellow solid; yield 64\% (365.0 mg, $0.94 \mathrm{mmol}$ ); $\mathrm{mp} 72-73{ }^{\circ} \mathrm{C} .{ }^{1} \mathrm{H}$ NMR $\left(400 \mathrm{MHz}, \mathrm{CDCl}_{3}\right): \delta(\mathrm{ppm}) 7.63(\mathrm{~d}, 1 \mathrm{H}), 7.36(\mathrm{~d}, 1 \mathrm{H}), 6.86-$ $6.82(\mathrm{~m}, 2 \mathrm{H}), 6.24(\mathrm{~d}, 1 \mathrm{H}), 5.47(\mathrm{td}, 1 \mathrm{H}), 5.10-5.07(\mathrm{~m}, 3 \mathrm{H})$, $4.60(\mathrm{~d}, 2 \mathrm{H}), 2.15-2.03(\mathrm{~m}, 8 \mathrm{H}), 1.99-1.95(\mathrm{~m}, 4 \mathrm{H}), 1.76$ (s, 3H), 1.67 (s, 3H), $1.60(\mathrm{~s}, 3 \mathrm{H}), 1.59(\mathrm{~s}, 3 \mathrm{H}), 1.58(\mathrm{~s}, 3 \mathrm{H})$.

5,7-Diacetyloxy-4-methyl-2H-chromen-2-one (5) 1 eq of the coumarin $\mathbf{3 b}$ and 2 eq of acetic anhydride were added to the appropriate amount of pyridine, and the mixture was heated at $80{ }^{\circ} \mathrm{C}$. After the completion of the reaction, pyridine was removed in vacuo and a solid product was obtained. The final pure coumarin was selected after recrystallization. Green solid; yield $72 \%$ (273.5 mg, $0.99 \mathrm{mmol})$; mp 143$144{ }^{\circ} \mathrm{C}$ (lit. m.p. $\left.150-151{ }^{\circ} \mathrm{C}\right)$ [42]. ${ }^{1} \mathrm{H}$ NMR $(600 \mathrm{MHz}$, $\left.\mathrm{CDCl}_{3}\right): \delta(\mathrm{ppm}) 7.06(\mathrm{~s}, 1 \mathrm{H}, \mathrm{H}-8), 6.87(\mathrm{~s}, 1 \mathrm{H}, \mathrm{H}-6), 6.20$ (s, $1 \mathrm{H}, \mathrm{H}-3), 2.50\left(\mathrm{~s}, 3 \mathrm{H}, 4-\mathrm{CH}_{3}\right), 2.37$ (s, 3H, 7- $\left.\mathrm{CH}_{3} \mathrm{CO}\right)$, $2.32\left(\mathrm{~s}, 3 \mathrm{H}, 5-\mathrm{CH}_{3} \mathrm{CO}\right)$.

\section{Cell culture}

Dulbecco's modified Eagle's medium (DMEM, D6429), foetal bovine serum (FBS, F9665), trypsin (T3924), L-glutamine (G7513), antibiotics (penicillin - streptomycin,
P0781) and (3-(4,5-dimethylthiazol-2-yl)-2,5-diphenyltetrazolium bromide) (MTT, M2128) were purchased from Sigma-Aldrich (Gillingham, UK). Dulbecco's modified Eagle's medium with no glucose (DMEM, A1443001) was purchased from Gibco Thermo Fisher Scientific, UK. Human breast adenocarcinoma cell lines MCF-7 (HTB-22 ${ }^{\mathrm{TM}}$ ) and MDA-MB-231 (HTB-26 ${ }^{\mathrm{TM}}$ ) were kindly donated from Manchester Cancer Research Labs (University of Manchester, UK). Human colon fibroblasts 18-Co (CRL-1459 ${ }^{\mathrm{TM}}$ ) were purchased from ATCC.

\section{General cell culture}

Unless otherwise specified, all cell culture experiments were performed in a humidified 5\% (v/v) $\mathrm{CO}_{2}$ air atmosphere at $37{ }^{\circ} \mathrm{C}$ in complete medium, and cell culture growth media were supplemented with $10 \%(\mathrm{v} / \mathrm{v})$ foetal bovine serum and $2 \mathrm{mM}$ L-glutamine. Human breast adenocarcinoma cell lines were cultured, maintained at densities lower than $1 \times 10^{6}$ cells $/ \mathrm{cm}^{2}$ and discarded upon reaching passage number 60 . Stromal healthy cells (human colorectal fibroblasts, $18 \mathrm{Co}$ ) were cultured using complete DMEM medium supplemented also with $1 \%(\mathrm{v} / \mathrm{v})$ penicillin-streptomycin. Cells were maintained at densities less than $1 \times 10^{6} \mathrm{cells} / \mathrm{cm}^{2}$ and discarded upon reaching passage number 12 .

\section{Nutrient-deprived conditions}

Cells were culture using nutrient-deprived cell culture conditions (i.e. cell culture media with no glucose, L-glutamine, HEPES and sodium pyruvate) to mimic conditions similar to the tumour microenvironment. Note that these experiments were performed only using breast cancer cells. MCF-7 and MDA-MB-231 cells were seeded in 96-well plates (Corning Inc., NY, USA) at a density of $1 \times 10^{4}$ and $6.7 \times 10^{3}$ cells/ $\mathrm{cm}^{2}$, respectively. Cells were incubated with coumarin derivatives at concentrations of $0.1,1,10,50,100$ and $250 \mu \mathrm{M}$ up to $48 \mathrm{~h}$. Untreated cells (negative) and cells incubated with $0.5 \%$ (v/v) DMSO in complete media (positive) were used as controls.

\section{Coumarins cytotoxicity}

The full library of coumarins was tested in both breast cancer cell lines, i.e. MCF-7 and MDA-MB-231. Fibroblast were used as "healthy" control. Stocks of compounds $\mathbf{3 a}-\mathbf{3 c}$, $\mathbf{4 a}-\mathbf{4 m}$ and $\mathbf{5}$ were dissolved in pure DMSO and then diluted in complete media. (Note that DMSO concentration was kept lower than $0.5 \%(\mathrm{v} / \mathrm{v})$.) Briefly, MCF-7 and MDA-MB-231 cells were seeded in 96-well plates (3799, Corning Inc., NY, USA) at a density of $1 \times 10^{4}$ and $6.7 \times 10^{3}$ cells $/ \mathrm{cm}^{2}$, respectively, whereas $18 \mathrm{Co}$ fibroblasts were seeded at a density of $1 \times 10^{4}$ cells $/ \mathrm{cm}^{2}$. Cells were incubated with coumarin 
derivatives at concentrations $0.1,1,10,50,100$ and 250 $\mu \mathrm{M}$ for $48 \mathrm{~h}$. Untreated cells (negative) and cells incubated with $0.5 \%$ (v/v) DMSO in complete media (positive) were used as controls.

\section{Cell metabolism assay}

For each treatment, cell viability was measured via MTT assay after 48-h incubation as following described. Cell culture medium was replaced with $150 \mu \mathrm{L}$ of fresh medium and $30 \mu \mathrm{L}$ of MTT solution, and cells were incubated for $4 \mathrm{~h}$ $\left(37^{\circ} \mathrm{C}, 5 \% \mathrm{CO}_{2}\right)$. After the formazan crystal formation, cell culture medium was removed from each well and replaced with $200 \mu \mathrm{L}$ of DMSO. The absorbance was measured at 540-nm wavelength using a plate reader (Synergy 2 Biotek plate reader, Gen5 software).

\section{Toxicity $\left(\mathrm{IC}_{50}\right)$ and identification of potent coumarins}

$\mathrm{IC}_{50}$ values were calculated (nonlinear regression, normalized response-variable slope) with GraphPad Prism (version 7.04). Values were ranked and classified as: high toxicity, moderate toxicity, poor toxicity and no toxicity. Threshold were set as 60,80 and $>100 \mu \mathrm{M}$, respectively. The selection was used in order to test only the most toxic coumarins under cell culture condition more relevant to the tumour microenvironment, i.e. deprived cell culture media (Sect. 3.3).

\section{Results and discussion}

\section{Design and synthesis of coumarin analogues}

Our previous results concerning the cytotoxic activity evaluation of natural oxyprenylated coumarins [14] in combination with the latest literature data led us to design, a new series of diprenyloxy as well as dialkyloxy coumarins. The new series were designed in order to evaluate the influence of the disubstitution as well as the length of the lipophilic chain in the cytotoxicity against breast cancer cell lines.

In order to efficiently synthesize the desired coumarin analogues and the naturally occurring oxyprenylated coumarins, the appropriate hydroxy-4-substituted coumarins 3a and $\mathbf{3 b}$ were firstly synthesized via Pechmann reaction using iron (III) chloride $\left(\mathrm{FeCl}_{3}\right)$ as the catalyst [36]. Compounds $\mathbf{3 a}$ and $\mathbf{3 b}$ as well as the commercially available 7-hydroxycoumarin (umbelliferone, $\mathbf{3 c}$ ) were subsequently alkylated with the appropriate commercially available alkyl bromide using potassium carbonate $\left(\mathrm{K}_{2} \mathrm{CO}_{3}\right)$ in acetone (Scheme 1). For the preparation of $\mathbf{4 m}$, the required geranylgeranyl iodide was prepared according to the method of AlvarezManzaneda et al. [37].

All the coumarin derivatives were purified using flash column chromatography and were structurally identified using ${ }^{1} \mathrm{H}$ and ${ }^{13} \mathrm{C}$ NMR spectroscopy and HRMS spectrometry.<smiles>[R]c1cc(O)cc(O)c1</smiles>

1a. $R_{1}=H$

1b. $\mathrm{R}_{1}=\mathrm{OH}$<smiles>[R]C(=O)CC(=O)OCC</smiles>

(a)

2a. $\mathrm{R}=\mathrm{nC}_{3} \mathrm{H}_{7}$ 2b. $\mathrm{R}=\mathrm{CH}_{3}$

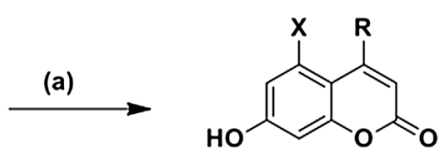

3a. $X=\mathrm{H}, \mathrm{R}=\mathrm{nC}_{3} \mathrm{H}_{7}$ 3b. $X=\mathrm{OH}, \mathrm{R}=\mathrm{CH}_{3}$ 3c. $X=H, R=H$

(c)

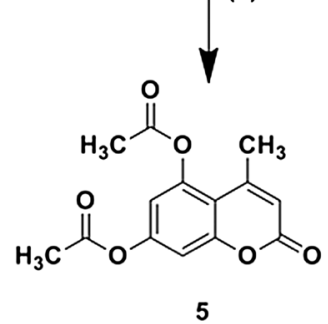

(b)<smiles>[R]Oc1cc([X])c2c([R])cc(=O)oc2c1</smiles>

4a. $\mathrm{X}=\mathrm{H}, \mathrm{R}=\mathrm{CH}_{3}, \mathrm{R}_{1}=$ prenyl 4b. $X=\mathrm{H}, \mathrm{R}=\mathrm{CH}_{3}, \mathrm{R}_{1}=$ geranyl 4c. $X=\mathrm{H}, \mathrm{R}=\mathrm{CH}_{3}, \mathrm{R}_{1}=$ farnesyl 4d. $X=\mathrm{H}, \mathrm{R}=\mathrm{CH}_{3}, \mathrm{R}_{1}=\mathrm{nC}_{8} \mathrm{H}_{17}$ 4e. $X=H, R=n C_{3} H_{7}, R_{1}=$ prenyl 4f. $X=\mathrm{H}, \mathrm{R}=\mathrm{nC}_{3} \mathrm{H}_{7}, \mathrm{R}_{1}=\mathrm{nC}_{8} \mathrm{H}_{17}$ 4g. $\mathrm{R}=\mathrm{CH}_{3}, \mathrm{R}_{1}=$ prenyl, $\mathrm{X}=\mathrm{O}$-prenyl 4h. $\mathrm{R}=\mathrm{nC}_{3} \mathrm{H}_{7}, \mathrm{R}_{1}=$ prenyl, $X=\mathrm{O}$-prenyl 4i. $\mathrm{R}=\mathrm{nC}_{3} \mathrm{H}_{7}, \mathrm{R}_{1}=$ geranyl, $X=\mathrm{O}$-geranyl 4j. $X=H, R=H, R_{1}=$ prenyl 4k. $X=H, R=H, R_{1}=$ geranyl 4l. $X=H, R=H, R_{1}=$ farnesyl 4m. $X=H, R=H, R_{1}=$ geranylgeranyl

Scheme 1 Synthesis of $\mathbf{3 a}, \mathbf{3 b}, \mathbf{4 a}-\mathbf{4 m}$ and 5; Reagents and conditions: $\mathbf{a} \mathrm{FeCl}_{3} / 70{ }^{\circ} \mathrm{C}, 80-90 \%, \mathbf{b} \mathrm{R}_{2} \mathrm{Br}$ or geranylgeranyl iodide, $\mathrm{K}_{2} \mathrm{CO}_{3}$, acetone, $50-60 \%$, $\mathbf{c ~} \mathrm{Ac}_{2} \mathrm{O}$, pyridine, $80^{\circ} \mathrm{C}$ 


\section{Cytotoxicity towards breast cancer cells}

The systematic variations on the alkyl chain as well as the position of substitution at the coumarin scaffold were examined as potential factors which could affect anticancer activity. The first set of experiments identified the most potent candidates among the coumarin derivatives herein synthesized. Cytotoxicity was firstly evaluated on two breast cancer cell lines: MCF-7 and MDA-MB-231. MCF-7 cells were selected as they retain several characteristics of differentiated mammary epithelium proliferation, as well as expressing oestrogen receptor, whereas MDA-MB-231 was selected as expressing a more aggressive and metastatic cells that do not express high levels of the oestrogen, progesterone or HER2 receptors (i.e. triple negative).

Coumarins were classified as possessing high $\left(\mathrm{IC}_{50}<80 \mu \mathrm{M}\right)$, moderate $\left(80 \mu \mathrm{M}<\mathrm{IC}_{50}<100 \mu \mathrm{M}\right)$ and poor toxicity $\left(100 \mu \mathrm{M}<\mathrm{IC}_{50}<250 \mu \mathrm{M}\right)$; compounds with $\mathrm{IC}_{50}$ values $>250 \mu \mathrm{M}$ were classified as non-toxic. Coumarin derivatives $\mathbf{4 c}, \mathbf{4 d}, \mathbf{4 g}, \mathbf{4 k}$ and $\mathbf{4 l}$ were identified as the topfive most potent compounds tested in this study (Table 1) and were selected for further investigation.

One of the main drawbacks of cytotoxic compounds is the poor selectivity towards cancer cells, with undesired effects on healthy cells, e.g. fibroblasts and epithelia. In an effort to understand whether coumarins have any effect on 'healthy' cells, human fibroblasts (18-Co) were treated with the most potent coumarin derivatives. As control, fibroblasts were also treated with a non-toxic coumarin (i.e. umbelliferone, 3c). Interestingly, cytotoxicity data evidenced no effect of the selected coumarins on fibroblasts (shown in Table 2) with the exception of coumarin $\mathbf{4 k}$ that showed some toxicity towards 'healthy' cells. The tested compounds were not as toxic as typical chemotherapeutics with $\mathrm{IC}_{50} \mathrm{~s}$ at the scale of few hundred $\mathrm{nM}$ such as doxorubicin [43-46] or gemcitabine [47-49], but they did appear to have no significant effects on 'healthy' fibroblasts as compared to the aforementioned chemo-agents. This is a very positive result in view of development of (nano)formulations and further translation of such compounds.

Auraptene (4j) was found to be the most potent compound among the library of coumarins tested in this study, confirming what has been already observed in other in vitro studies and in various in vivo animal models [50]. Its effect on cancer cells is still not clear and could be associated with induction of carcinogen-detoxifying enzymes, inhibition of free radical generation or metalloproteinase production [51]. The length of the prenyl chain seems to affect the activity of the compounds: auraptene ( $\mathbf{4 k}, 10$ carbons) is more potent compared to its prenyloxy analogue $(\mathbf{4} \mathbf{j}, 5$ carbons) against the tested breast cancer cells. However, umbelliprenin (41, 15 carbons) and coumarin $\mathbf{4 m}$ (20 carbons) exhibited lower antitumour potency, with umbelliprenin being more toxic than coumarin $\mathbf{4 m}$ in both cancer cell lines (Fig. 1).

The number of substituents on the aromatic ring could also play a role in the activity: 5,7-diprenyloxy-4-methylcoumarin (4g) is approximately 2.5 times more cytotoxic compared to its monosubstituted analogue (compound 4a) against MCF-7 cells. Increasing the chain length of the substituents, as in 7-geranylgeranyloxy-coumarin $(\mathbf{4 n})$, results in complete loss of activity against both cell lines (Fig. 1, Table 1).

In an effort to better investigate the role of unsaturation on cytotoxicity, the coumarin analogues $\mathbf{4 d}$ and $\mathbf{4 f}$, which possess a saturated alkyloxy substituent, were synthesized. Only coumarin $\mathbf{4 d}$ exhibited a moderate potency against MCF7 cells, whereas only a slight toxic effect was observed on MDA-MB-231 (Table 1); moreover, no toxicity was observed on fibroblasts (Table 2). These results suggest that this specific modification can participate in different biochemical pathways compared to unsaturated substituents; however, further research is necessary to confirm this and identify specific pathways.

Finally, we investigated the activity of derivatives as function of lipophilicity through the introduction of a methyl group at position 4 of the coumarin scaffold. The methylated coumarin derivatives $\mathbf{4 b}, \mathbf{4 a}$ and $\mathbf{4 c}$ exhibited a rather moderate cytotoxic effect compared to their corresponded nonmethylated analogues $\mathbf{4 k}, \mathbf{4 j}$ and $\mathbf{4 l}$. This observation suggests that substitution at position 4 might not directly link to increased toxicity. Furthermore, comparing the compounds with different substitutions at position4 (e.g. coumarins $\mathbf{4 d}$ vs $\mathbf{4 f}$, coumarins $\mathbf{4 g}$ vs $\mathbf{4 h}$ ) increased toxicity was observed in both cancer cell lines for compounds with methyl substitution (Fig. 1).

\section{Cytotoxicity in tumour relevant in vitro models}

In accordance with the work of Jun et al. [52] and Devji et al. [41], we were motivated to assess the activity of some selected derivatives under nutrient-deprived conditions (NDCs). Cancer cells are programmed in a non-ordinary way to exhibit high glycolytic activity even under sufficient aerobic conditions [53]. In hypoxic tumour conditions, when oxygen depletion and low vascularization take place, cancer cells often find the way to proliferate rapidly by foregoing oxidative phosphorylation and instead ferment large 
Table $1 \quad \mathrm{IC}_{50}$ values $(\mu \mathrm{M})$ of coumarin derivatives (18 compounds) on MCF-7 and MDA-MB-231 breast cancer cells obtained after 48-h incubation

\begin{tabular}{|c|c|c|c|}
\hline $\begin{array}{l}\text { Coumarin } \\
\text { derivative }\end{array}$ & Chemical structure & $\begin{array}{c}\text { MCF }-7 \\
\mathrm{IC}_{50} \text { values }(\mu \mathrm{M})\end{array}$ & $\begin{array}{l}\text { MDA-MB-231 } \\
\text { IC }_{50} \text { values }(\mu \mathrm{M})\end{array}$ \\
\hline $3 a$ & & $225 \pm 19$ & $206 \pm 9$ \\
\hline $\mathbf{3 b}$ & & $>250$ & $>250$ \\
\hline $3 c$ & & $>250$ & $>250$ \\
\hline $4 a$ & & $179 \pm 35$ & $169 \pm 23$ \\
\hline $4 b$ & & $129 \pm 13$ & $137 \pm 9$ \\
\hline $4 c$ & & $105 \pm 3$ & $110 \pm 29$ \\
\hline 4d & & $82 \pm 19$ & $113 \pm 7$ \\
\hline
\end{tabular}


Table 1 (continued)

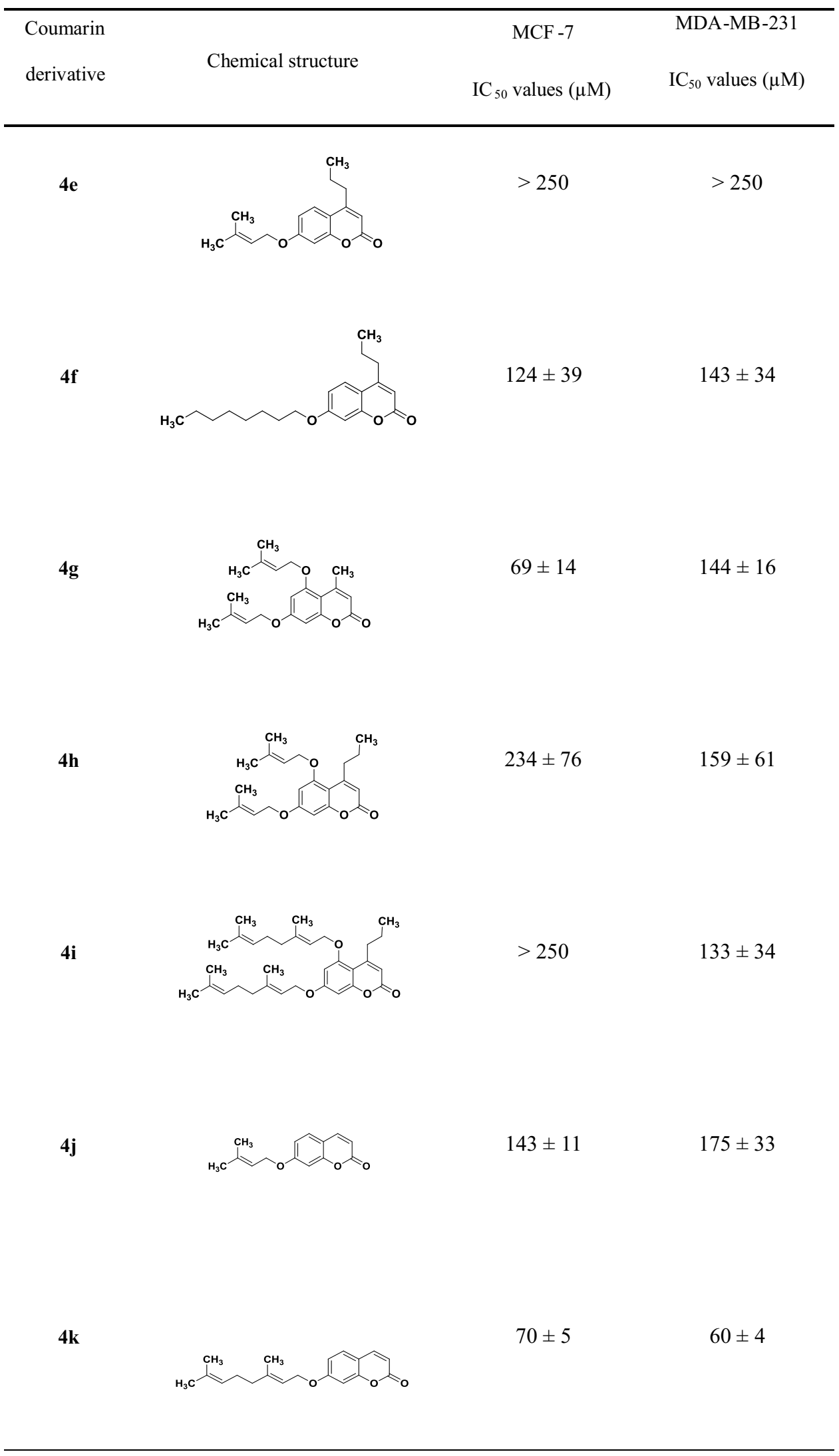


Table 1 (continued)

\begin{tabular}{|c|c|c|c|}
\hline Coumarin & & MCF -7 & MDA-MB-231 \\
\hline derivative & Chemical structure & $\mathrm{IC}_{50}$ values $(\mu \mathrm{M})$ & $\mathrm{IC}_{50}$ values $(\mu \mathrm{M})$ \\
\hline
\end{tabular}

41<smiles>CC(C)=CCC/C(C)=C/CC/C(C)=C/COc1ccc2ccc(=O)oc2c1</smiles>

$4 m$

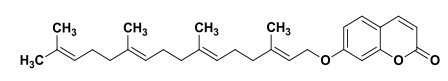

5

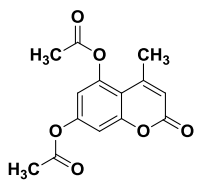

$98 \pm 9$

$88 \pm 3$
$>250$

$187 \pm 66$

$>250$

$>250$

Data are expressed as mean \pm SD. of two independent experiments ( $n=3$ samples for each experiment). As control, MCF-7 and MDA-231 were incubated with $[0.001-50] \mu \mathrm{g} / \mathrm{mL}$ of doxorubicin for $48 \mathrm{~h}$, reporting $\mathrm{IC}_{50}$ values of $(0.97 \pm 0.60) \mu \mathrm{M}$ and $(0.48 \pm 0.14) \mu \mathrm{M}$, respectively

amounts of glucose into lactate under aerobic glycolysis, known as the Warburg effect [54, 55]. Moreover, hypoxia tends to boost this phenomenon by up-regulating the HIF-1a factor that "switches on" glycolytic and glucose transporter gene expression [56]. Breast carcinoma cell lines behave in a glucose-dependent manner and derive the majority of energy needed from high-throughput glycolysis [56, 57]. Hyperglycaemic systemic conditions, i.e. diabetes, have been proved to further promote the migratory invasiveness of breast malignancies in patients [55].

On that basis, we exposed the breast cancer cells to nutrient-deprived conditions where culture media were supplemented only with $2.5 \%$ v/v FBS, but not additional glucose, L-glutamine, sodium pyruvate. The coumarins tested were auraptene (4k), umbelliprenin (4l) and analogues 4d, 4c and 4g. As shown in Table 3, the tested compounds showed selective preferential cytotoxicity under nutrient-deprived conditions with umbelliprenin (4I) to be the most potent candidate as its pharmacological activity was remarkably enhanced by 15 times $\left(\mathrm{IC}_{50}=9.0\right.$ and 7.0 for MCF7 and MDA-MB231 cells, respectively). In similar studies, Zhang et al. and Jun et al. reported the high preferential cytotoxicity of umbelliprenin (4I) and its C6 analogue under NDC against pancreatic cancer cells $[52,58]$. It should be therefore noted that these derivatives could represent a potential new tool for treating aggressively metastatic hypoxic tumours. The exact mechanism of action, though, should be further investigated. 
Table $2 \mathrm{IC}_{50}$ values $(\mu \mathrm{M})$ of the most potent coumarin derivatives (five compounds: $\mathbf{4 c}, \mathbf{4 d}, \mathbf{4 g}, \mathbf{4 k}$ and $\mathbf{4 l}$ ) on $18 \mathrm{Co}$ colon fibroblasts (healthy control)

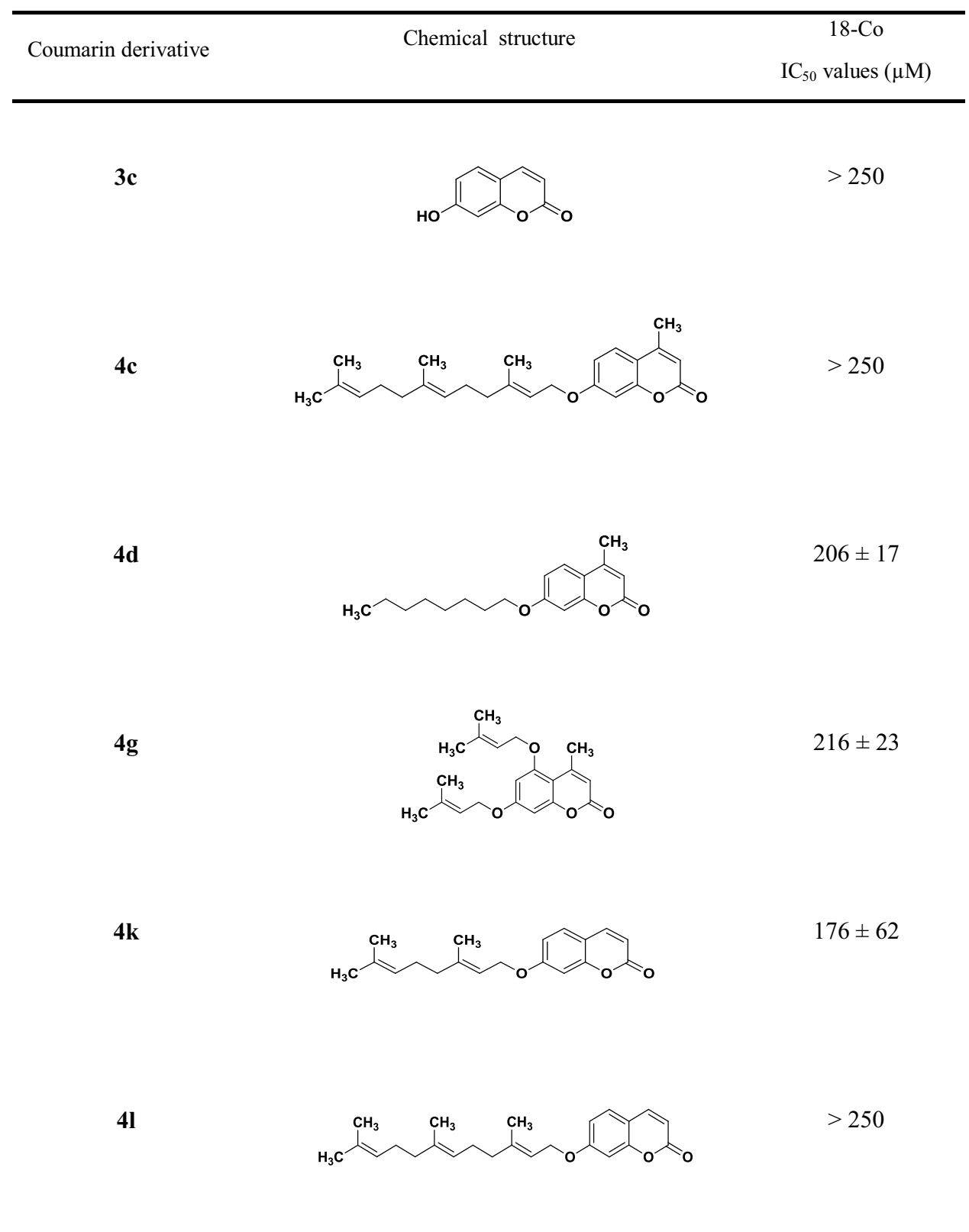

Data are expressed as mean \pm SD and are obtained from $n=2$ independent experiments 
Fig. 1 Effect of substituents on coumarins on MCF-7 cells: cell viability was measured after 48-h incubation with different concentrations of coumarins and $\mathrm{IC}_{50}$ values determined using nonlinear regression (GraphPad Prism, v7). Coumarins were compared on the basis of the following structural features: a length of lipophilic chain, coumarin $\mathbf{4 k}$ versus $\mathbf{4 j}$ (10 vs 5 carbons) and coumarin $4 \mathbf{l}$ versus $4 \mathbf{m}$ (15 vs 20 carbons); b position of the substituent on the coumarin scaffold, coumarin $\mathbf{4 g}$ vs $\mathbf{4 a} ; \mathbf{c}$ effect of the saturation degree (substitution at position C7), coumarin 4d vs $\mathbf{4 f} ; \mathbf{d}$ the presence of methyl group (substitution at position C4), coumarin $\mathbf{4 b}$ versus $\mathbf{4 k}$ and coumarin $\mathbf{4 a}$ versus $\mathbf{4 j}$ (data not shown), coumarin $\mathbf{4 c}$ versus $\mathbf{4 l}$ (data not shown); e the presence of propyl group (substitution at position $\mathrm{C} 4$ ), coumarins $\mathbf{4 g}$ versus $\mathbf{4 h}$ and coumarins $\mathbf{4 d}$ versus 4f (data not shown)

\section{A I Length of lipophilic chain}

$\mathbf{4 j}$ vs $4 \mathbf{k}$

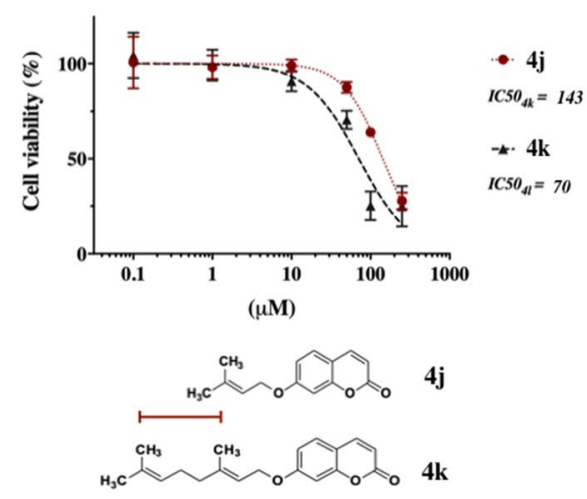

B | Group substitution

$4 a$ vs $4 g$
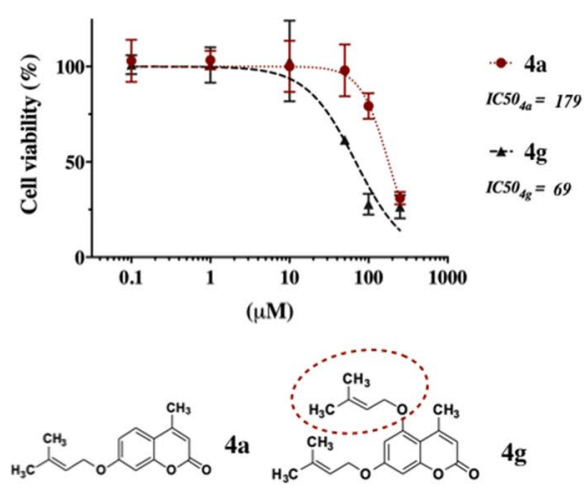

D I Substitution in 3' carbon

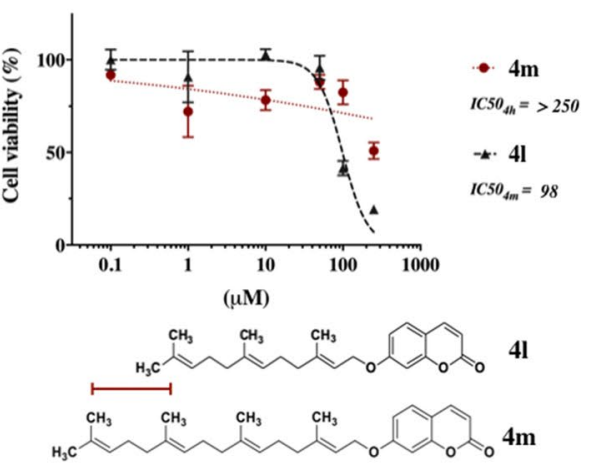

C I Saturation degree in 7' carbon

4d vs $4 f$
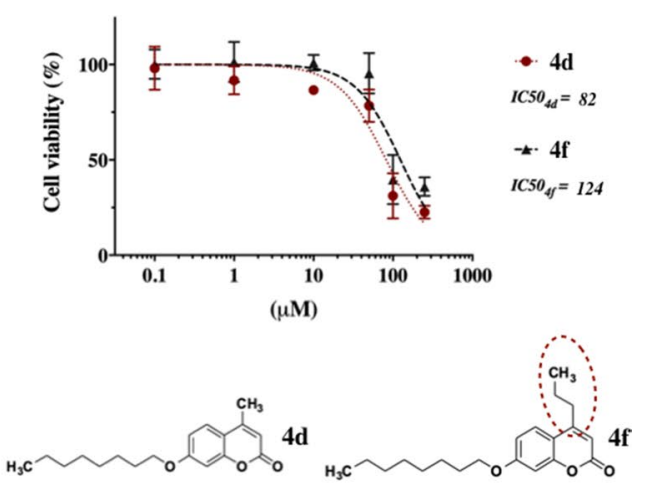

E I Alkylation in 3' carbon

$4 g$ vs $4 h$
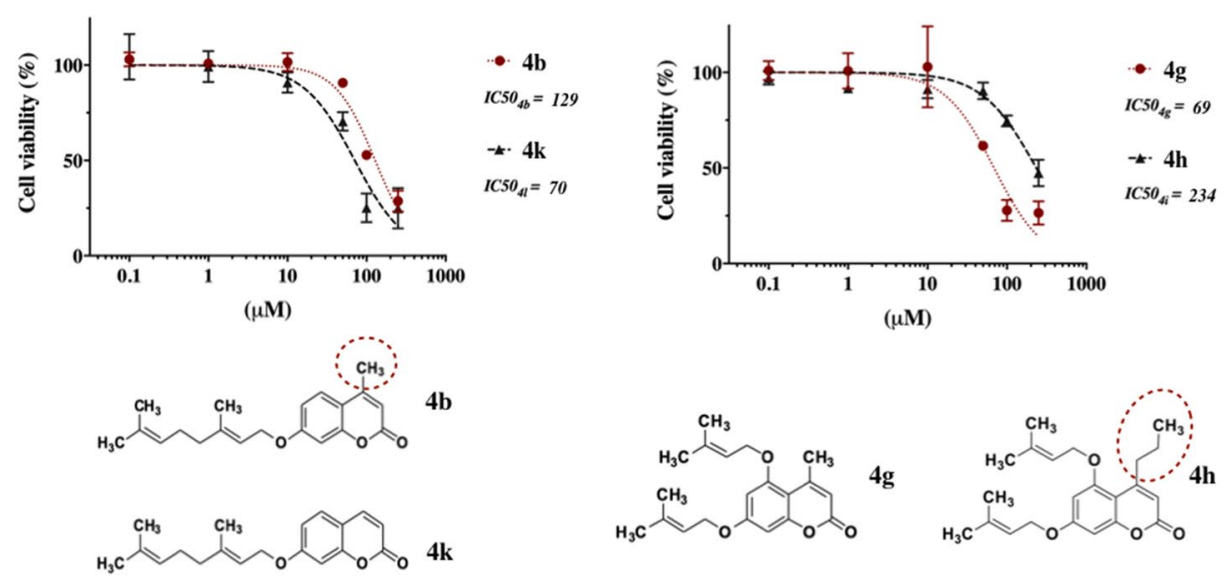
Table $3 \quad \mathrm{IC}_{50}$ values $(\mu \mathrm{M})$ of the tested coumarin derivatives $\mathbf{4 c}, \mathbf{4 d}, \mathbf{4 g}, \mathbf{4 k}$ and $\mathbf{4 l}$. Values were obtained for both MCF-7 and MDA-MB-231 breast cancer cells cultured under nutrient-deprived conditions and incubated with coumarin derivatives up to $48 \mathrm{~h}$

\begin{tabular}{|c|c|c|c|}
\hline $\begin{array}{l}\text { Coumarin } \\
\text { derivative }\end{array}$ & Chemical structure & $\begin{array}{c}\mathrm{MCF}-7 \\
\mathrm{IC}_{50} \text { values } \\
(\mu \mathrm{M})\end{array}$ & $\begin{array}{c}\text { MDA-MB-231 } \\
\text { IC }_{50} \text { values } \\
(\mu \mathrm{M})\end{array}$ \\
\hline $4 c$ & & $19 \pm 3$ & $51 \pm 9$ \\
\hline $4 d$ & & $29 \pm 3$ & $63 \pm 5$ \\
\hline $4 g$ & & $35 \pm 9$ & $11 \pm 2$ \\
\hline $4 k$ & & $14 \pm 5$ & $12 \pm 8$ \\
\hline 41 & & $9 \pm 1$ & $7 \pm 2$ \\
\hline
\end{tabular}

Data are expressed as average $\pm \mathrm{SD}$ of $n=3$ independent experiments

\section{Conclusions}

A series of novel alkoxy-coumarin derivatives were synthesized and tested for their cytotoxicity against the MCF7 and MDA-MB-231 breast cancer cells. The results of this study indicate that alkylation modification induces noticeable differentiation in pharmacological activity of coumarins. Auraptene (4k) was found to possess the most potent cytotoxic activity among the tested derivatives followed by compounds $\mathbf{4 c}, \mathbf{4 d}, \mathbf{4 g}$ and $\mathbf{4 l}$. The tested compounds seemed not to affect the cell viability of the healthy $18 \mathrm{Co}$ fibroblasts but for the highest dose only. The amplification of the cytotoxic effect of the above pharmacophores under nutrient-deprived conditions, with umbelliprenin (4l) being the lead compound, indicates that these compounds could lead to potential new therapeutics for highly metastatic hypoxic tumours once their mechanisms are fully understood.
Acknowledgements Eleni Kavetsou gratefully acknowledges financial support from State Scholarships Foundation (IKY): The realization of the doctoral dissertation was co-funded by the "Grant Scheme for Postgraduate Studies in Secondary Education" of the Operational Program "Human Resource Development, Education and Lifelong Learning" of the NSRF 2014-2020 with the co-financing of the European Social Fund. Leonidas Gkionis is indebted to EPSRC for a Ph.D. studentship as part of the Graphene NOWNANO Doctoral Training Centre.

Funding Not applicable.

Availability of data and material (data transparency) The raw data supporting the conclusions of this manuscript will be made available by the authors, without undue reservation, to any qualified researcher.

\section{Compliance with ethical standards}

Conflict of interest The authors declare that they have no conflict of interest.

Ethics approval (include appropriate approvals or waivers) Not applicable. 
Open Access This article is licensed under a Creative Commons Attribution 4.0 International License, which permits use, sharing, adaptation, distribution and reproduction in any medium or format, as long as you give appropriate credit to the original author(s) and the source, provide a link to the Creative Commons licence, and indicate if changes were made. The images or other third party material in this article are included in the article's Creative Commons licence, unless indicated otherwise in a credit line to the material. If material is not included in the article's Creative Commons licence and your intended use is not permitted by statutory regulation or exceeds the permitted use, you will need to obtain permission directly from the copyright holder. To view a copy of this licence, visit http://creativecommons.org/licenses/by/4.0/.

\section{References}

1. Marcom PK (2017) Breast cancer. In: Ginsburg G, Willard H, David S (eds) Genomic and precision medicine: primary care, 3rd edn. Elsevier Ltd, pp 181-194

2. Tao ZQ, Shi A, Lu C et al (2015) Breast cancer: epidemiology and etiology. Cell Biochem Biophys 72:333-338. https://doi. org/10.1007/s12013-014-0459-6

3. Hanahan D, Weinberg RA (2011) Hallmarks of cancer: the next generation. Cell 144:646-674

4. Guerra AR, Duarte MF, Duarte IF (2018) Targeting tumor metabolism with plant-derived natural products: emerging trends in cancer therapy. J Agric Food Chem 66:10663-10685. https://doi. org/10.1021/acs.jafc.8b04104

5. Lee KW, Bode AM, Dong Z (2011) Molecular targets of phytochemicals for cancer prevention. Nat Rev Cancer 11:211-218

6. Zhang L, Xu Z (2019) Coumarin-containing hybrids and their anticancer activities. Eur J Med Chem 181:111587

7. Fayed EA, Sabour R, Harras MF, Mehany ABM (2019) Design, synthesis, biological evaluation and molecular modeling of new coumarin derivatives as potent anticancer agents. Med Chem Res 28:1284-1297. https://doi.org/10.1007/s00044-019-02373-x

8. Kaur M, Kohli S, Sandhu S et al (2015) Coumarin: a promising scaffold for anticancer agents. Anticancer Agents Med Chem 15:1032-1048. https://doi.org/10.2174/18715206156661501011 25503

9. O'Kennedy R, Thornes DR (1997) Coumarins: biology, applications and mode of action. Wiley, Chichester

10. Detsi A, Kontogiorgis C, Hadjipavlou-Litina D (2017) Coumarin derivatives: an updated patent review (2015-2016). Expert Opin Ther Pat 27:1201-1226

11. Stefanachi A, Leonetti F, Pisani L et al (2018) Coumarin: a natural, privileged and versatile scaffold for bioactive compounds. Molecules 23:1-34

12. Barot KP, Jain SV, Kremer L et al (2015) Recent advances and therapeutic journey of coumarins: current status and perspectives. Med Chem Res 24:2771-2798

13. Venugopala KN, Rashmi V, Odhav B (2013) Review on natural coumarin lead compounds for their pharmacological activity. Biomed Res Int 2013:1-14

14. Kavetsou E, Gkionis L, Galani G et al (2017) Synthesis of prenyloxy coumarin analogues and evaluation of their antioxidant, lipoxygenase (LOX) inhibitory and cytotoxic activity. Med Chem Res 26:856-866. https://doi.org/10.1007/s0004 4-017-1800-6

15. Roussaki M, Zelianaios K, Kavetsou E et al (2014) Structural modifications of coumarin derivatives: determination of antioxidant and lipoxygenase (LOX) inhibitory activity. Bioorganic Med Chem 22:6586-6594. https://doi.org/10.1016/j.bmc.2014.10.008

16. Al-Majedy YK, Al-Duhaidahawi DL, Al-Azawi KF et al (2016) Coumarins as potential antioxidant agents complemented with suggested mechanisms and approved by molecular modeling studies. Molecules 21:2-11. https://doi.org/10.3390/molecules2 1020135

17. Tamene D, Endale M (2019) Antibacterial activity of coumarins and carbazole alkaloid from roots of clausena anisata. Adv Pharmacol Sci 2019:1-8. https://doi.org/10.1155/2019/5419854

18. Yang L, Wu L, Yao X et al (2018) Hydroxycoumarins: new, effective plant-derived compounds reduce Ralstonia pseudosolanacearum populations and control tobacco bacterial wilt. Microbiol Res 215:15-21. https://doi.org/10.1016/j.micres.2018.05.011

19. Tian D, Wang F, Duan M et al (2019) Coumarin analogues from the Citrus grandis (L.) osbeck and their hepatoprotective activity. J Agric Food Chem 67:1937-1947. https://doi.org/10.1021/ acs.jafc. 8 b06489

20. Berrino E, Milazzo L, Micheli L et al (2019) Synthesis and evaluation of carbonic anhydrase inhibitors with carbon monoxide releasing properties for the management of rheumatoid arthritis. J Med Chem 62:7233-7249. https://doi.org/10.1021/acs.jmedc hem.9b00845

21. Zhao YL, Yang XW, Wu BF et al (2019) Anti-inflammatory effect of Pomelo peel and its bioactive coumarins. J Agric Food Chem 67:8810-8818. https://doi.org/10.1021/acs.jafc.9b02511

22. Peperidou A, Bua S, Bozdag M et al (2018) Novel 6- and 7-substituted coumarins with inhibitory action against lipoxygenase and tumor-associated carbonic anhydrase IX. Molecules 23:13-17. https://doi.org/10.3390/molecules23010153

23. Zhao H, Donnelly AC, Kusuma BR et al (2011) Engineering an antibiotic to fight cancer: optimization of the novobiocin scaffold to produce anti-proliferative agents. J Med Chem 54:3839-3853. https://doi.org/10.1021/jm200148p

24. Herrera-R A, Castrillón W, Otero E et al (2018) Synthesis and antiproliferative activity of 3- and 7-styrylcoumarins. Med Chem Res 27:1893-1905. https://doi.org/10.1007/s00044-018-2202-0

25. Farley CM, Dibwe DF, Ueda JY et al (2016) Evaluation of synthetic coumarins for antiausterity cytotoxicity against pancreatic cancers. Bioorganic Med Chem Lett 26:1471-1474. https://doi. org/10.1016/j.bmcl.2016.01.054

26. Ricci F, Carrassa L, Christodoulou MS et al (2018) A highthroughput screening of a chemical compound library in ovarian cancer stem cells. Comb Chem High Throughput Screen 21:50-56. https://doi.org/10.2174/13862073216661801240 93406

27. Cao D, Liu Y, Yan W et al (2016) Design, synthesis, and evaluation of in vitro and in vivo anticancer activity of 4-substituted coumarins: a novel class of potent tubulin polymerization inhibitors. J Med Chem 59:5721-5739. https://doi.org/10.1021/ acs.jmedchem.6b00158

28. Fiorito S, Epifano F, Taddeo VA, Genovese S (2018) Recent acquisitions on oxyprenylated secondary metabolites as antiinflammatory agents. Eur J Med Chem 153:116-122. https:// doi.org/10.1016/j.ejmech.2017.08.038

29. Alhassan AM, Abdullahi MI, Uba A, Umar A (2014) Prenylation of aromatic secondary metabolites: a new frontier for development of novel drugs. Trop J Pharm Res 13:307-314

30. Hasan M, Genovese S, Fiorito S et al (2017) Oxyprenylated phenylpropanoids bind to MT1 melatonin receptors and inhibit breast cancer cell proliferation and migration. J Nat Prod 80:3324-3329. https://doi.org/10.1021/acs.jnatprod.7b00853

31. Okuyama S, Minami S, Shimada N et al (2013) Anti-inflammatory and neuroprotective effects of auraptene, a citrus coumarin, following cerebral global ischemia in mice. Eur J Pharmacol 699:118-123. https://doi.org/10.1016/j.ejphar.2012.11.043

32. Okuyama S, Semba T, Toyoda N et al (2016) Auraptene and other prenyloxyphenylpropanoids suppress microglial activation and dopaminergic neuronal cell death in a 
lipopolysaccharide-induced model of Parkinson's disease. Int J Mol Sci 17:1-11. https://doi.org/10.3390/ijms 17101716

33. Iranshahi M, Askari M, Sahebkar A, Hadjipavlou-Litina D (2009) Evaluation of antioxidant, anti-inflammatory and lipoxygenase inhibitory activities of the prenylated coumarin umbelliprenin. Daru 17:99-103

34. Yan H, Ma Z, Peng S, Deng X (2013) Anti-inflammatory effect of auraptene extracted from trifoliate orange (Poncirus trifoliate) on LPS-stimulated RAW 264.7 cells. Inflammation 36:1525-1532. https://doi.org/10.1007/s10753-013-9695-y

35. La VD, Zhao L, Epifano F et al (2013) Anti-inflammatory and wound healing potential of citrus auraptene. J Med Food 16:961-964. https://doi.org/10.1089/jmf.2013.0029

36. Prousis KC, Avlonitis N, Heropoulos GA, Calogeropoulou T (2014) $\mathrm{FeCl} 3$-catalysed ultrasonic-assisted, solvent-free synthesis of 4-substituted coumarins. A useful complement to the Pechmann reaction. Ultrason Sonochem 21:937-942. https:// doi.org/10.1016/j.ultsonch.2013.10.018

37. Alvarez-Manzaneda EJ, Chahboun R, Cabrera Torres E et al (2005) Reaction of allylic and benzylic alcohols and esters with PPh 3/I2: one-pot synthesis of $\beta, \gamma$-unsaturated compounds. Tetrahedron Lett 46:3755-3759. https://doi.org/10.1016/j.tetle t.2005.03.132

38. Takeuch N, Kasama T, Aida Y et al (1991) Pharmacological activities of the prenylcoumarins, developed from folk usage as a medicine of Peucedanum japonicum thunb. Chem Pharm Bull 39:1415-1421. https://doi.org/10.1248/cpb.39.1415

39. Row EC, Brown SA, Stachulski AV, Lennard MS (2006) Design, synthesis and evaluation of furanocoumarin monomers as inhibitors of CYP3A4. Org Biomol Chem 4:1604-1610. https://doi. org/10.1039/b601096b

40. Askari M, Sahebkar A, Iranshahi M (2009) Synthesis and purification of 7-prenyloxycoumarins and herniarin as bioactive natural coumarins. Iran J Basic Med Sci 12:63-69. https://doi. org/10.22038/ijbms.2009.5145

41. Devji T, Reddy C, Woo C et al (2011) Pancreatic anticancer activity of a novel geranylgeranylated coumarin derivative. Bioorganic Med Chem Lett 21:5770-5773. https://doi. org/10.1016/j.bmcl.2011.08.005

42. Canter FW, Curd FH, Robertson A (1931) Hydroxy-carbonyl compounds. Part III. The preparation of coumarins and 1:4:-benzopyrones from phloroglucinol and resorcinol. J Chem Soc. https://doi.org/10.1039/jr9310001255

43. Wen Sh, Su Sc, Liou Bh et al (2018) Sulbactam-enhanced cytotoxicity of doxorubicin in breast cancer cells. Cancer Cell Int 18:1-18. https://doi.org/10.1186/s12935-018-0625-9

44. Arif IS, Hooper CL, Greco F et al (2013) Increasing doxorubicin activity against breast cancer cells using PPAR $\gamma$-ligands and by exploiting circadian rhythms. Br J Pharmacol 169:1178-1188. https://doi.org/10.1111/bph.12202

45. Fang XJ, Jiang H, Zhu YQ et al (2014) Doxorubicin induces drug resistance and expression of the novel CD44st via NF- $\kappa$ B in human breast cancer MCF-7 cells. Oncol Rep 31:2735-2742. https://doi.org/10.3892/or.2014.3131

46. You Y, Xu Z, Chen Y (2018) Doxorubicin conjugated with a trastuzumab epitope and an MMP-2 sensitive peptide linker for the treatment of HER2-positive breast cancer. Drug Deliv 25:448-460. https://doi.org/10.1080/10717544.2018.1435746

47. Tao XM, Wang JC, Wang JB et al (2012) Enhanced anticancer activity of gemcitabine coupling with conjugated linoleic acid against human breast cancer in vitro and in vivo. Eur $\mathrm{J}$ Pharm Biopharm 82:401-409. https://doi.org/10.1016/j. ejpb.2012.06.007

48. Li A, Fridley B, Kalari K et al (2008) Gemcitabine and cytosine arabinoside cytotoxicity: association with lymphoblastoid cell expression. Cancer Res 68:7050-7058. https://doi. org/10.1158/0008-5472.CAN-08-0405

49. Hernández-Vargas H, Rodríguez-Pinilla SM, Julián-Tendero M et al (2007) Gene expression profiling of breast cancer cells in response to gemcitabine: NF- $\mathrm{BB}$ pathway activation as a potential mechanism of resistance. Breast Cancer Res Treat 102:157-172. https://doi.org/10.1007/s10549-006-9322-9

50. Krishnan P, Yan KJ, Windler D et al (2009) Citrus auraptene suppresses cyclin D1 and significantly delays N-methyl nitrosourea induced mammary carcinogenesis in female Sprague-Dawley rats. BMC Cancer 9:1-12. https://doi. org/10.1186/1471-2407-9-259

51. Krishnan P, Kleiner-Hancock H (2012) Effects of Auraptene on IGF-1 stimulated cell cycle progression in the human breast cancer cell line, MCF-7. Int J Breast Cancer 2012:1-8. https:// doi.org/10.1155/2012/502092

52. Jun M, Bacay AF, Moyer J et al (2014) Synthesis and biological evaluation of isoprenylated coumarins as potential anti-pancreatic cancer agents. Bioorganic Med Chem Lett 24:4654-4658. https://doi.org/10.1016/j.bmcl.2014.08.038

53. Izuishi K, Kato K, Ogura T et al (2000) Remarkable tolerance of tumor cells to nutrient deprivation: possible new biochemical target for cancer therapy. Cancer Res 60:6201-6207

54. Zhuang Y, Chan DK, Haugrud AB, Miskimins WK (2014) Mechanisms by which low glucose enhances the cytotoxicity of metformin to cancer cells both in vitro and in vivo. PLoS ONE. https://doi.org/10.1371/journal.pone.0108444

55. Takatani-Nakase T, Matsui C, Maeda S et al (2014) High glucose level promotes migration behavior of breast cancer cells through zinc and its transporters. PLoS ONE 9:e90136. https:// doi.org/10.1371/journal.pone.0090136

56. O'Mahony F, Razandi M, Pedram A et al (2012) Estrogen modulates metabolic pathway adaptation to available glucose in breast cancer cells. Mol Endocrinol 26:2058-2070. https:// doi.org/10.1210/me.2012-1191

57. Simões RV, Serganova IS, Kruchevsky N et al (2015) Metabolic plasticity of metastatic breast cancer cells: adaptation to changes in the microenvironment. Neoplasia 17:671-684. https ://doi.org/10.1016/j.neo.2015.08.005

58. Zhang H, Zhou R, Jun M et al (2016) Identification of the factors responsible for the selective in vitro cytotoxic activity of isoprenylated coumarin derivatives under nutrient-deprived conditions. J Cancer 7:160-166. https://doi.org/10.7150/jca.13243

Publisher's Note Springer Nature remains neutral with regard to jurisdictional claims in published maps and institutional affiliations. 